\title{
Partonic coalescence in relativistic heavy ion collisions
}

\author{
V. Greco and C. M. Ko \\ Cyclotron Institute and Physics Department, Texas A\&M University, College Station, Texas 77843-3366, USA \\ P. Lévai \\ KFKI Research Institute for Particle and Nuclear Physics, P.O. Box 49, Budapest 1525, Hungary
}

(Received 9 May 2003; published 5 September 2003)

\begin{abstract}
Using a covariant coalescence model, we study hadron production in relativistic heavy ion collisions from both soft partons in the quark-gluon plasma and hard partons in minijets. Including transverse flow of soft partons and independent fragmentation of minijet partons, the model is able to describe available experimental data on pion, kaon, and antiproton spectra. The resulting antiproton to pion ratio is seen to increase at low transverse momenta and reaches a value of about 1 at intermediate transverse momenta, as observed in experimental data at RHIC. A similar dependence of the antikaon to pion ratio on transverse momentum is obtained, but it reaches a smaller value at intermediate transverse momenta. At high transverse momenta, the model predicts that both the antiproton to pion and the antikaon to pion ratio decrease and approach those given by the perturbative QCD. Both collective flow effect and coalescence of minijet partons with partons in the quark-gluon plasma affect significantly the spectra of hadrons with intermediate transverse momenta. Elliptic flows of phi mesons and baryons such as protons, lambdas, cascades, and omegas have also been evaluated from partons with elliptic flows extracted from fitting measured pion and kaon elliptic flows. The predicted proton and lambda elliptic flows are consistent with available experimental data.
\end{abstract}

DOI: 10.1103/PhysRevC.68.034904

PACS number(s): 25.75.Dw, 25.75.Nq, 12.38.Bx

\section{INTRODUCTION}

Recently, there is a renewed interest in using the parton coalescence or recombination model to understand hadron production from the quark-gluon plasma (QGP) expected to be formed in relativistic heavy ion collisions [1,2,4-7]. Emphases in these studies are, however, different from earlier ones based on the coalescence model such as the ALCOR [8] and the MICOR [9] model. Instead of addressing particle yields and their ratios, these new studies were more concerned with observables related to collective dynamics and production of hadrons with relatively large transverse momentum. In Ref. [1], the parton coalescence was used to convert the quark matter, which is formed from melted soft strings produced in initial soft collisions, to hadrons. Including parton coalescence in a multiphase transport model (AMPT) [10], it was found that partonic effects are important for describing measured large elliptic flows and narrow twopion correlation functions at RHIC. In Refs. [2,3], it was shown that hadron production based on parton coalescence is able to account for the qualitative difference between the observed elliptic flows of mesons and baryons. Based on parton recombination, a parton transverse momentum distribution was obtained in Ref. [4] from the measured pion spectrum and was then used to predict the kaon and proton transverse momentum spectra. The parton coalescence model was further found in Refs. $[5,6]$ to be able to explain the observed enhancement of intermediate transverse momentum protons and antiprotons. While coalescence of partons from a quarkgluon plasma with high effective temperature was considered in Ref. [5], their coalescence with high transverse momentum minijet partons, which are produced from initial hard scatterings, was introduced in Ref. [6] as a new mechanism for hadronization of minijet partons.
Besides independent fragmentations to hadrons as usually considered, minijet partons produced in relativistic heavy ion collisions [11] were allowed in Ref. [6] to coalesce with partons from the quark-gluon plasma expected to be formed in relativistic heavy ion collisions, as suggested in Ref. [7] for studying the flavor ordering of the elliptic flows of hadrons with intermediate transverse momenta. Since minijet partons have a power-law transverse momentum spectrum while partons in the quark-gluon plasma have an exponential thermal spectrum, this mechanism leads to an enhanced production of hadrons with intermediate transverse momentum. Because of stronger enhancement for baryons and antibaryons than for pions from this hadronization mechanism, a large antiproton to pion ratio of about 1 is obtained at intermediate transverse momenta as seen in the experimental data from the PHENIX collaboration [12]. However, in this study hadrons from hadronization of the quark-gluon plasma are taken to also have exponential thermal spectra extending to all transverse momenta. Furthermore, in order to obtain a semianalytical expression for the coalescence formula, only comoving partons at zero rapidity are considered in evaluating the coalescence probability for hadron production. Although relativistic kinematics was used in this study, the model is not fully covariant. In the present paper, we relax these simplifications by using a covariant coalescence model and treating more generally parton coalescence via a Monte Carlo method. Also, we include coalescence among partons from the quark-gluon plasma so that hadrons with low momenta are treated on the same footing as those with intermediate momenta. We show that this improved coalescence model for partons from both quark-gluon plasma and minijets is able to reproduce the experimental transverse momentum spectra of pions, antikaons, and antiprotons measured at RHIC as well as the antiproton to pion ratio as functions of 
transverse momentum. We further predict the dependence of the $K^{-} / \pi^{-}$ratio on transverse momentum. Fitting quark elliptic flows to measured pion and kaon elliptic flows, the predicted elliptic flows of protons and $\Lambda$ are found to agree with available experimental data. The $\Xi$ and $\Omega$ elliptic flows are also predicted and are smaller than that of $\Lambda$ as a result of smaller elliptic flow for light quarks than strange quarks. Similarly, the predicted $\phi$ meson elliptic flow is smaller than that of kaons.

The paper is organized as follows. In Sec. II, we describe the general formalism of a covariant coalescence model for mesons and baryons. How minijet and quark-gluon plasma partons are determined for $\mathrm{Au}+\mathrm{Au}$ collisions at $200 \mathrm{~A} \mathrm{GeV}$ is described in Sec. III. In Sec. IV, the Monte Carlo method used for treating coalescence among partons whose numbers vary by many orders of magnitude is presented. Results for the transverse momentum spectra of pions, antiprotons, and antikaons obtained from the coalescence model are given in Sec. V. Ratios of the antiproton to pion and the antikaon to pion transverse momentum spectra are also shown. We further study the effect of coalescence of minijet partons with those in the quark-gluon plasma. The effect of collective flow on the spectra and ratios of produced hadrons is studied in Sec. VI. In Sec. VII, the elliptic flow of hadrons based on that of quarks is also studied. Finally, we conclude in Sec. VIII with a summary of present work and an outlook about future developments and applications of the parton coalescence model.

\section{THE COALESCENCE MODEL}

Using the covariant coalescence model of Dover et al. [13], the number of mesons formed from the coalescence of quark and antiquarks can be written as

$$
\begin{aligned}
N_{M}= & g_{M} \int p_{1} \cdot d \sigma_{1} p_{2} \cdot d \sigma_{2} \frac{d^{3} \mathbf{p}_{1}}{(2 \pi)^{3} E_{1}} \frac{d^{3} \mathbf{p}_{2}}{(2 \pi)^{3} E_{2}} \\
& \times f_{q}\left(x_{1} ; p_{1}\right) f_{q}^{-}\left(x_{2} ; p_{2}\right) f_{M}\left(x_{1}, x_{2} ; p_{1}, p_{2}\right) .
\end{aligned}
$$

In the above, $d \sigma$ denotes an element of a spacelike hypersurface. The functions $f_{q}(x, p)$ and $f_{q}^{-}(x, p)$ are, respectively, covariant distribution functions of quarks and antiquarks in the phase space, and they are normalized to their numbers, i.e.,

$$
\int p \cdot d \sigma \frac{d^{3} \mathbf{p}}{(2 \pi)^{3} E} f_{q, \bar{q}}(x, p)=N_{q, \bar{q}} .
$$

Other factors in Eq. (1) describe the dynamic process of converting a quark and an antiquark to a bound state meson in the presence of a partonic matter. The statistical factor $g_{M}$ takes into account the internal quantum numbers in forming a colorless meson from spin- $-\frac{1}{2}$ color quark and antiquark. For mesons considered here, i.e., $\pi, \rho, K$, and $K^{*}$, the statistical factors are $g_{\pi}=g_{K}=1 / 36$ and $g_{\rho}=g_{K^{*}}=1 / 12$. The coalescence probability function $f_{M}\left(x_{1}, x_{2} ; p_{1}, p_{2}\right)$ depends on the overlap of the quark and antiquark wave functions with the wave function of the meson as well as the interactions of emitted virtual partons, which are needed for balancing the energy and momentum, with the partonic matter. Neglecting the off-shell effects and taking the wave functions of quark and antiquark to be plane waves, the coalescence probability function is then simply the covariant meson Wigner distribution function. Since the constituent quark model describes reasonably the properties of hadrons, the hadron Wigner function is thus approximately a Gaussian function in $x_{1}$ $-x_{2}$ and $p_{1}-p_{2}$. Here, we take it to have a uniform distribution as in Ref. [6], i.e.,

$$
\begin{aligned}
f_{M}\left(x_{1}, x_{2} ; p_{1}, p_{2}\right)= & \frac{9 \pi}{2\left(\Delta_{x} \Delta_{p}\right)^{3}} \Theta\left(\Delta_{x}^{2}-\left(x_{1}-x_{2}\right)^{2}\right) \Theta\left(\Delta_{p}^{2}\right. \\
& \left.-\frac{1}{4}\left(p_{1}-p_{2}\right)^{2}+\frac{1}{4}\left(m_{1}-m_{2}\right)^{2}\right),
\end{aligned}
$$

where $\Delta_{x}$ and $\Delta_{p}$ are the covariant spatial and momentum coalescence radii, and they are related by the uncertainty relation $\Delta_{x} \cdot \Delta_{p} \approx 1$. The factors in front of $\Theta$ functions are introduced to obtain the correct normalization for the meson Wigner function in the nonrelativistic limit, i.e., $\int d^{3} \mathbf{x} d^{3} \mathbf{p} f_{M}(\mathbf{x}, \mathbf{p})=(2 \pi)^{3}$. Here we have used $\hbar=c=1$.

For ultrarelativistic heavy ion collisions at RHIC, it is convenient to introduce rapidity variables $y$ and $\eta$ in the momentum and the coordinate space, respectively, and these are defined by

$$
y=\frac{1}{2} \ln \frac{E+p_{z}}{E-p_{z}}, \quad \eta=\frac{1}{2} \ln \frac{t+z}{t-z} .
$$

Using these variables, the spatial coordinate becomes $x$ $=\left(\tau \cosh \eta,-\tau \sinh \eta,-\mathbf{r}_{\mathrm{T}}\right)$, with $\tau=\sqrt{t^{2}-z^{2}}$ and $\mathbf{r}_{\mathrm{T}}$ denoting, respectively, the proper time and transverse coordinates; while the momentum is $p=\left(m_{\mathrm{T}} \cosh y,-m_{\mathrm{T}} \sinh y,-\mathrm{p}_{\mathrm{T}}\right)$ with $m_{\mathrm{T}}=\sqrt{m_{q}^{2}+\mathbf{p}_{\mathrm{T}}^{2}}$ being the transverse mass in terms of the quark mass $m_{q}$ and its transverse momentum $\mathbf{p}_{\mathrm{T}}$. The momentum volume element is then

$$
\frac{d^{3} \mathbf{p}}{E}=d y d^{2} \mathbf{p}_{\mathrm{T}},
$$

while the spatial volume element becomes

$$
p \cdot d \sigma=\tau m_{\mathrm{T}} \cosh (y-\eta) d \eta d^{2} \mathbf{r}_{\mathrm{T}},
$$

if we adopt a hypersurface of constant longitudinal proper times.

The quark and antiquark phase space distribution functions are then given by

$$
f_{q, \bar{q}}(x, p)=\frac{(2 \pi)^{3}}{\tau m_{\mathrm{T}} \cosh (y-\eta)} \frac{d N_{q, \bar{q}}}{d \eta d^{2} \mathbf{r}_{\mathrm{T}} d y d^{2} \mathbf{p}_{\mathrm{T}}},
$$

and the yield of mesons from coalescence of quarks and antiquarks is 


$$
\begin{aligned}
N_{M}= & g_{M} \int d \eta_{1} d^{2} \mathbf{r}_{1 \mathrm{~T}} d \eta_{2} d^{2} \mathbf{r}_{2 \mathrm{~T}} d y_{1} d^{2} \mathbf{p}_{1 \mathrm{~T}} d y_{2} d^{2} \mathbf{p}_{2 \mathrm{~T}} \\
& \times \frac{d N_{q}}{d \eta_{1} d^{2} \mathbf{r}_{1 \mathrm{~T}} d y_{1} d^{2} \mathbf{p}_{1 \mathrm{~T}}} \frac{d N_{q}}{d \eta_{2} d^{2} \mathbf{r}_{2 \mathrm{~T}} d y_{2} d^{2} \mathbf{p}_{2 \mathrm{~T}}} \\
& \times f_{M}\left(x_{1}, x_{2} ; p_{1}, p_{2}\right) .
\end{aligned}
$$

The transverse momentum spectrum of mesons can be obtained from Eq. (8) by multiplying the right hand side with

$$
1=\int d^{2} \mathbf{p}_{\mathrm{T}} \delta^{(2)}\left(\mathbf{p}_{\mathrm{T}}-\mathbf{p}_{1 \mathrm{~T}}-\mathbf{p}_{2 \mathrm{~T}}\right),
$$

and then differentiating both sides of the equation with respect to $\mathbf{p}_{\mathrm{T}}$. The resulting meson transverse momentum spectrum from quark and antiquark coalescence is

$$
\begin{aligned}
\frac{d N_{M}}{d^{2} \mathbf{p}_{\mathrm{T}}}= & g_{M} \int d \eta_{1} d^{2} \mathbf{r}_{1 \mathrm{~T}} d \eta_{2} d^{2} \mathbf{r}_{2 \mathrm{~T}} d y_{1} d^{2} \mathbf{p}_{1 \mathrm{~T}} d y_{2} d^{2} \mathbf{p}_{2 \mathrm{~T}} \\
& \times \frac{d N_{q}}{d \eta_{1} d^{2} \mathbf{r}_{1 \mathrm{~T}} d y_{1} d^{2} \mathbf{p}_{1 \mathrm{~T}}} \frac{d N_{q}^{-}}{d \eta_{2} d^{2} \mathbf{r}_{2 \mathrm{~T}} d y_{2} d^{2} \mathbf{p}_{2 \mathrm{~T}}} \\
& \times f_{M}\left(x_{1}, x_{2} ; p_{1}, p_{2}\right) \delta^{(2)}\left(\mathbf{p}_{\mathrm{T}}-\mathbf{p}_{1 \mathrm{~T}}-\mathbf{p}_{2 \mathrm{~T}}\right) .
\end{aligned}
$$

For quarks and antiquarks produced at central rapidities in relativistic heavy ion collisions, it is reasonable to assume that their longitudinal momentum distributions are boostinvariant, i.e., independent of rapidity. Furthermore, these are expected to satisfy the Bjorken correlation of equal spatial and momentum rapidities, i.e., $\eta=y$. The quark and antiquark phase space distribution functions in the rapidity range $\Delta y$ can then be expressed as

$$
\frac{d N_{q, \bar{q}}}{d \eta d^{2} \mathbf{r}_{\mathrm{T}} d y d^{2} \mathbf{p}_{\mathrm{T}}}=\left.\frac{\delta(\eta-y)}{\Delta y} \frac{d N_{q, \bar{q}}}{d^{2} \mathbf{r}_{\mathrm{T}} d^{2} \mathbf{p}_{\mathrm{T}}}\right|_{|y| \leqslant \Delta y / 2} .
$$

This leads to the following meson transverse momentum spectrum from coalescence of quarks and antiquarks:

$$
\begin{aligned}
\frac{d N_{M}}{d^{2} \mathbf{p}_{\mathrm{T}}}= & \frac{g_{M}}{(\Delta y)^{2}} \int d^{2} \mathbf{r}_{1 \mathrm{~T}} d^{2} \mathbf{r}_{2 \mathrm{~T}} d^{2} \mathbf{p}_{1 \mathrm{~T}} d^{2} \mathbf{p}_{2 \mathrm{~T}} \\
& \times\left.\left.\frac{d N_{q}}{d^{2} \mathbf{r}_{1 \mathrm{~T}} d^{2} \mathbf{p}_{1 \mathrm{~T}}}\right|_{\left|y_{1}\right| \leqslant \Delta y / 2} \frac{d N_{q}^{-}}{d^{2} \mathbf{r}_{2 \mathrm{~T}} d^{2} \mathbf{p}_{2 \mathrm{~T}}}\right|_{\left|y_{2}\right| \leqslant \Delta y / 2} \\
& \times \int d \eta_{1} d y_{1} d \eta_{2} d y_{2} \delta\left(\eta_{1}-y_{1}\right) \delta\left(\eta_{2}-y_{2}\right) \\
& \times f_{M}\left(x_{1}, x_{2} ; p_{1}, p_{2}\right) \delta^{(2)}\left(\mathbf{p}_{\mathrm{T}}-\mathbf{p}_{1 \mathrm{~T}}-\mathbf{p}_{2 \mathrm{~T}}\right) .
\end{aligned}
$$

The above result can be simplified if there is no correlation between parton transverse momenta and positions, such as in the absence of collective transverse flow, and if partons are also uniformly distributed in the transverse space. In this case, the quark and antiquark distributions only depend on transverse momentum, i.e.,

$$
\left.\frac{d N_{q, \bar{q}}}{d^{2} \mathbf{r}_{\mathrm{T}} d^{2} \mathbf{p}_{\mathrm{T}}}\right|_{|y| \leqslant \Delta y / 2}=\left.\frac{1}{\pi R_{\perp}^{2}} \frac{d N_{q, \bar{q}}}{d^{2} \mathbf{p}_{\mathrm{T}}}\right|_{|y| \leqslant \Delta y / 2},
$$

where $R_{\perp}$ is the transverse radius of the system. Considering a small rapidity range such as $\Delta y \leqslant 1$, we have

$$
\begin{aligned}
\left(x_{1}-x_{2}\right)^{2} & =2 \tau^{2}\left[1-\cosh \left(\eta_{1}-\eta_{2}\right)\right]-\left(\mathbf{r}_{1 \mathrm{~T}}-\mathbf{r}_{2 \mathrm{~T}}\right)^{2} \\
& \approx-\tau^{2}\left(\eta_{1}-\eta_{2}\right)^{2}-\left(\mathbf{r}_{1 \mathrm{~T}}-\mathbf{r}_{2 \mathrm{~T}}\right)^{2} \approx-\left(\mathbf{r}_{1}-\mathbf{r}_{2}\right)^{2}
\end{aligned}
$$

and

$$
\begin{aligned}
\left(p_{1}-p_{2}\right)^{2}= & m_{1 \mathrm{~T}}^{2}+m_{2 \mathrm{~T}}^{2}-2 m_{1 \mathrm{~T}} m_{2 \mathrm{~T}} \cosh \left(y_{1}-y_{2}\right) \\
& -\left(\mathbf{p}_{1 \mathrm{~T}}-\mathbf{p}_{2 \mathrm{~T}}\right)^{2} \approx\left(m_{1 \mathrm{~T}}-m_{2 \mathrm{~T}}\right)^{2}-\left(\mathbf{p}_{1 \mathrm{~T}}-\mathbf{p}_{2 \mathrm{~T}}\right)^{2} .
\end{aligned}
$$

After carrying out the integrals in transverse space, the meson transverse momentum spectra are given by

$$
\begin{aligned}
\frac{d N_{M}}{d^{2} \mathbf{p}_{\mathrm{T}}}= & g_{M} \frac{6 \pi}{\tau \Delta y R_{\perp}^{2} \Delta_{p}^{3}} \int d^{2} \mathbf{p}_{1 \mathrm{~T}} d^{2} \mathbf{p}_{2 \mathrm{~T}} \\
& \times\left.\left.\frac{d N_{q}}{d^{2} \mathbf{p}_{1 \mathrm{~T}}}\right|_{\left|y_{1}\right| \leqslant \Delta y / 2} \frac{d N_{q}^{-}}{d^{2} \mathbf{p}_{2 \mathrm{~T}}}\right|_{\left|y_{2}\right| \leqslant \Delta y / 2} \\
& \times \delta^{(2)}\left(\mathbf{p}_{\mathrm{T}}-\mathbf{p}_{1 \mathrm{~T}}-\mathbf{p}_{2 \mathrm{~T}}\right) \Theta\left(\Delta_{p}^{2}-\frac{1}{4}\left(\mathbf{p}_{1 \mathrm{~T}}-\mathbf{p}_{2 \mathrm{~T}}\right)^{2}\right. \\
& \left.-\frac{1}{4}\left[\left(m_{1 \mathrm{~T}}-m_{2 \mathrm{~T}}\right)^{2}-\left(m_{1}-m_{2}\right)^{2}\right]\right) .
\end{aligned}
$$

This result for meson transverse momentum spectra reduces to that used in our previous schematic study [6] if we assume that only comoving quarks and antiquarks can coalesce to hadrons and replace the argument of the $\Theta$ function in Eq. (16) by $\Delta_{p}^{2}$ minus the quark and antiquark relative momentum in meson rest frame. Since we will include collective transverse flow of partons in the quark-gluon plasma, Eq. (12) will be used in the following study.

To generalize the results for mesons to formation of baryons and antibaryons from the parton distribution functions, we take the baryon coalescence probability function as

$$
\begin{aligned}
& f_{B}\left(x_{1}, x_{2}, x_{3} ; p_{1}, p_{2}, p_{3}\right) \\
& =\frac{9 \pi}{2 \Delta_{x}^{3} \Delta_{p}^{3}} \Theta\left(\Delta_{x}^{2}-\frac{1}{2}\left(x_{1}-x_{2}\right)^{2}\right) \\
& \quad \times \Theta\left(\Delta_{p}^{2}-\frac{1}{2}\left(p_{1}-p_{2}\right)^{2}\right) \frac{9 \pi}{2 \Delta_{x}^{3} \Delta_{p}^{3}} \\
& \quad \times \Theta\left(\Delta_{x}^{2}-\frac{1}{6}\left(x_{1}+x_{2}-2 x_{3}\right)^{2}\right) \\
& \quad \times \Theta\left(\Delta_{p}^{2}-\frac{1}{6}\left[\left(p_{1}+p_{2}-2 p_{3}\right)^{2}\right.\right.
\end{aligned}
$$




$$
\left.\left.-\left(m_{1}+m_{2}-2 m_{3}\right)^{2}\right]\right),
$$

where we have taken for simplicity the same space and momentum coalescence radii for the relative Jacobi coordinates among three quarks. The normalization of $f_{B}$ is similar to that for meson coalescence probability function $f_{M}$, i.e., $(2 \pi)^{6}$ after integrating over the relative coordinates and momenta in the nonrelativistic limit.

For boost-invariant dynamics with Bjorken spatial and momentum rapidities correlation, we obtain the following baryon transverse momentum spectrum from coalescence of three quarks:

$$
\begin{aligned}
\frac{d N_{B}}{d^{2} \mathbf{p}_{\mathrm{T}}}= & \left.\frac{g_{B}}{(\Delta y)^{3}} \int \prod_{i=1}^{3} d^{2} \mathbf{r}_{i \mathrm{~T}} d^{2} \mathbf{p}_{i \mathrm{~T}} \frac{d N_{q}}{d^{2} \mathbf{r}_{i \mathrm{~T}} d^{2} \mathbf{p}_{i \mathrm{~T}}}\right|_{\left|y_{i}\right| \leqslant \Delta y / 2} \\
& \times \int \prod_{i=1}^{3} d \eta_{i} d y_{i} \delta\left(\eta_{i}-y_{i}\right) \\
& \times f_{B}\left(x_{1}, x_{2}, x_{3} ; p_{1}, p_{2}, p_{3}\right) \delta^{(2)} \\
& \times\left(\mathbf{p}_{\mathrm{T}}-\sum_{i=1}^{3} \mathbf{p}_{i \mathrm{~T}}\right) .
\end{aligned}
$$

In the above, $g_{B}$ is the statistical factor for formation of a baryon from three quarks. For baryons and antibaryons considered in present study, i.e., $p, \Delta, \bar{p}$ and $\bar{\Delta}$, the statistical factors are $g_{p}=g_{\bar{p}}=1 / 108$ and $g_{\Delta}=g_{\bar{\Delta}}=1 / 54$. The above formula can also be used for antibaryons by replacing quark momentum spectra by the momentum spectra of antiquarks.

\section{PARTON DISTRIBUTIONS}

We consider central $\mathrm{Au}+\mathrm{Au}$ collisions $(0-10 \%)$ at $200 A \mathrm{GeV}$ available at RHIC. In these collisions, hard processes between initial nucleons lead to production of minijet partons with large transverse momentum. Although minijet partons are produced promptly, it still takes a few $\mathrm{fm} / c$ for them to traverse the surrounding dense matter before converting to hadrons. Observed quenching of high transverse momentum hadrons in these collisions seems to indicate that the dense matter is the quark-gluon plasma expected to be formed from soft processes in the collisions. In this section, both parton momentum spectra in minijets and in the quarkgluon plasma are introduced.

\section{A. Minijet partons}

Partons at high transverse momenta (usually greater than $2 \mathrm{GeV}$ ) are mainly produced from initial hard collisions among nucleons. The transverse momentum distribution of these minijet partons in the midrapidity can be obtained from an improved perturbative QCD calculation [14]. It is given by $d N_{\text {jet }} / d^{2} p_{T}=1 / \sigma_{\text {tot }}^{0-10} d \sigma_{\text {jet }} / d^{2} p_{T}$ in terms of $\sigma_{\text {tot }}^{0-10}$ corresponding to the total cross section at central $10 \%$ of the collisions and the jet production cross section from nucleusnucleus collisions,

$$
\begin{aligned}
\frac{d \sigma_{\mathrm{jet}}}{d^{2} \mathbf{p}_{\mathrm{T}}}= & \int d^{2} \mathbf{b} d^{2} \mathbf{r} t_{\mathrm{Au}}(\mathbf{r}) t_{\mathrm{Au}}(\mathbf{b}-\mathbf{r}) \sum_{a b} \int d x_{a} d x_{b} d^{2} \mathbf{k}_{\mathbf{a T}} d^{2} \mathbf{k}_{\mathbf{b T}} \\
& \times g\left(\mathbf{k}_{\mathrm{aT}}\right) g\left(\mathbf{k}_{\mathbf{b T}}\right) f_{a / \mathrm{Au}}\left(x_{a}, Q^{2}\right) f_{b / \mathrm{Au}}\left(x_{b}, Q^{2}\right) \\
& \times \frac{\hat{s}}{\pi} \delta(\hat{s}+\hat{t}+\hat{u}) \frac{d \sigma^{a b}}{d \hat{t}} .
\end{aligned}
$$

In the above, $t_{\mathrm{A}}(\mathbf{r})$ is the thickness function of $\mathrm{Au}$ at transverse radius $\mathbf{r}$ given by integrating the nuclear density distribution along the longitudinal direction. The parton distribution function in a nucleon in the nucleus $\mathrm{Au}$ is denoted by $f_{a / \mathrm{Au}}\left(x, Q^{2}\right)$ including transverse smearing $g\left(\mathbf{k}_{\mathrm{T}}\right)$. The cross section $d \sigma^{a b} / d \hat{t}$ is the parton scattering cross section. Kinematic details and a systematic analysis of $p p$ collisions can be found in Ref. [14]. Using the GRV94 LO result for the parton distribution function [15] and the Kniehl-KramerPötter (KKP) fragmentation function from Ref. [16], measured data in the reaction $p p \rightarrow \pi^{0} X$ at $\sqrt{s}=200 \mathrm{GeV}$ can be reproduced with $Q=0.75 p_{\mathrm{T}}$ and $\left\langle k_{\mathrm{T}}^{2}\right\rangle=2 \mathrm{GeV}^{2}$.

In heavy ion collisions at RHIC, minijet partons are expected to lose energy by radiating soft partons as they traverse through the quark-gluon plasma. This effect can be taken into account by lowering their transverse momenta by the energy loss $\Delta E$, which depends on both the parton energy $E$ and an effective opacity parameter $L / \lambda$ according to the Gyulassy-Lévai-Vitev model [17].

Taking as momentum cutoff $p_{0}=2 \mathrm{GeV}$ for minijet partons and using an effective opacity $L / \lambda=3.5$ as extracted from fitting the spectrum of high transverse momentum pions measured at RHIC $[17,18]$, the transverse momentum spectra of minijet partons at midrapidity $(y=0)$ in central $\mathrm{Au}+\mathrm{Au}$ collisions at $\sqrt{s}=200 \mathrm{~A} \mathrm{GeV}$ are shown in Fig. 1 for gluons (short-dashed curve), $u$ and $d$ (solid curve), $\bar{u}$ and $\bar{d}$ (dashed curve) as well as $s$ and $\bar{s}$ (dash-dotted curve) quarks. These spectra can be parametrized as

$$
\frac{d N_{\mathrm{jet}}}{d^{2} \mathbf{p}_{\mathrm{T}}}=A\left(\frac{B}{B+p_{\mathrm{T}}}\right)^{n} .
$$

Values for the parameters $A, B$, and $n$ for gluons, light and strange quarks and antiquarks are given in Table I. For later calculation of parton coalescence probability, masses of minijet partons are taken to be the current quark masses, i.e., $10 \mathrm{MeV}$ for light quarks and $175 \mathrm{MeV}$ for strange quarks. Also, we assume that the rapidity distribution of minijet partons is uniform in the rapidity range of $y \in(-0.5,+0.5)$ considered in the present study.

\section{B. The quark-gluon plasma}

For partons in the quark-gluon plasma, their transverse momentum spectra are taken to have an exponential form. For their longitudinal momentum distribution, we assume that these are boost-invariant, i.e., these have a uniform rapidity distribution in the range $y \in(-0.5,+0.5)$. To take into account collective flow of quark-gluon plasma, these 


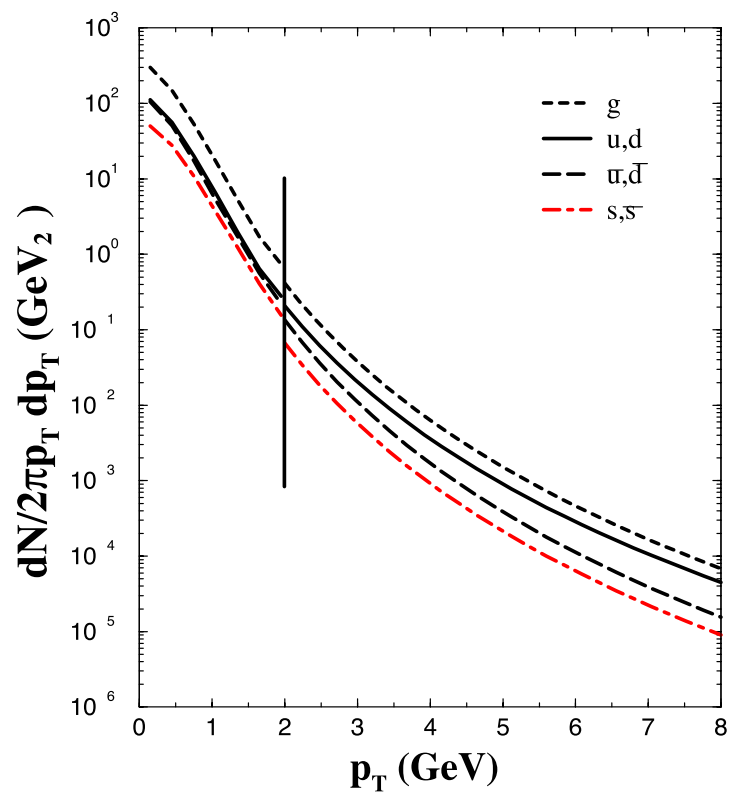

FIG. 1. (Color online) Transverse momentum distributions of partons at hadronization in $\mathrm{Au}+\mathrm{Au}$ collisions at $\sqrt{s}=200 \mathrm{~A} \mathrm{GeV}$ for gluons (short-dashed curve), $u$ and $d$ (solid curve), $\bar{u}$ and $\bar{d}$ (dashed curve) as well as $s$ and $\bar{s}$ (dash-dotted curve) quarks. Minijet partons have transverse momenta greater than $2 \mathrm{GeV}$, while partons from the quark-gluon plasma have transverse momenta below 2 $\mathrm{GeV}$.

partons are boosted by a flow velocity $\mathbf{v}_{\mathrm{T}}=\beta_{0}\left(\mathbf{r}_{\mathrm{T}} / R_{\perp}\right)$, depending on their transverse radial positions $r_{\mathrm{T}}$. Here, $R_{\perp}$ is the transverse size of the quark-gluon plasma at hadronization, and $\beta_{0}$ is the collective flow velocity of the quark-gluon plasma and is taken to be $0.5 c$. In this case, the light quarks and antiquarks transverse momentum spectra are given by

$$
\frac{d N_{q, \bar{q}}}{d^{2} \mathbf{r}_{\mathrm{T}} d^{2} \mathbf{p}_{\mathrm{T}}}=\frac{g_{q, \bar{q}} \tau m_{\mathrm{T}}}{(2 \pi)^{3}} \exp \left(-\frac{\gamma_{\mathrm{T}}\left(m_{\mathrm{T}}-\mathbf{p}_{\mathrm{T}} \cdot \mathbf{v}_{\mathrm{T}}\right) \mp \mu_{b}}{T}\right),
$$

where $g_{q}=g_{q}=6$ are spin-color degeneracies of light quarks and antiquarks, and the minus and plus signs are for quarks and antiquarks, respectively. The inverse slope parameter $T$ is taken to be $T=170 \mathrm{MeV}$, consistent with the phase transition temperature $(T \sim 165-185 \mathrm{MeV})$ from lattice QCD calculations. Masses of light quarks and antiquarks are taken to be $m_{q}=m_{q}^{-}=300 \mathrm{MeV}$, similar to the masses of constituent quarks due to possible nonperturbative effects in the

TABLE I. Parameters for minijet parton distributions given in Eq. (20) at midrapidity from $\mathrm{Au}+\mathrm{Au}$ at $\sqrt{s}=200 \mathrm{GeV}$.

\begin{tabular}{lccc}
\hline \hline & $A\left[1 / \mathrm{GeV}^{2}\right]$ & $B[\mathrm{GeV}]$ & $n$ \\
\hline$g$ & $3.2 \times 10^{4}$ & 0.5 & 7.1 \\
$u, d$ & $9.8 \times 10^{3}$ & 0.5 & 6.8 \\
$\bar{u}, \bar{d}$ & $1.9 \times 10^{4}$ & 0.5 & 7.5 \\
$s, \bar{s}$ & $6.5 \times 10^{3}$ & 0.5 & 7.4 \\
\hline \hline
\end{tabular}

quark-gluon plasma near hadronization [19]. For the quark baryon chemical potential $\mu_{b}$, we use a value of $\mu_{b}$ $=10 \mathrm{MeV}$ to give a light antiquark to quark ratio of 0.89 , which would then lead to an antiproton to proton ratio of about $(0.89)^{3}=0.7$, consistent with the observed ratio at midrapidity in heavy ion collisions at RHIC. The transverse flow effect is taken into account through the factor $\gamma_{\mathrm{T}}$ $=1 / \sqrt{1-v_{\mathrm{T}}^{2}}$.

The momentum spectra for strange quarks and antiquarks are similar to Eq. (21) with $m_{q}$ replaced by the strange quark mass $m_{s}=m_{s}^{-}=475 \mathrm{MeV}$ and $\mu_{b}$ replaced by $\mu_{b}-\mu_{s}$. The strange chemical potential $\mu_{s}$ is taken to be zero in order to have same strange and antistrange quarks numbers. The resulting strange quark to light quark ratio is then about 0.27 . Including contribution from decays of $\rho$ and $K^{*}$, this would then give a final $K^{-} / K^{+}=0.89$ and $K^{-} / \pi^{-}=0.24$, comparable to experimental data at midrapidity from RHIC. Equation (21) also applies to gluons after replacing $g_{q}$ by the gluon spin-color degeneracy $g_{g}=16$ and dropping the chemical potential. For the gluon mass, we take it to be similar to that of light quarks in order to take into account nonperturbative effects in the quark-gluon plasma.

Although the shape of quark-gluon plasma is nearly cylindrically symmetric, its parton momentum distribution is azimuthally asymmetric as shown by the finite elliptic flow of hadrons observed in experiments. Since we are interested in hadron spectra that are integrated over the azimuthal angle, including such asymmetry is not expected to change much our results. Nonetheless, the Monte Carlo method to be described in Sec. IV to evaluate the coalescence formula can be easily extended to take into account such effects in order to study the relation between the elliptic flow of partons and those of hadrons as suggested in Ref. [2] and to be studied in Sec. VII.

The quark-gluon plasma is further assumed to have a transverse radius of $R_{\perp}=8.3 \mathrm{fm}$ at proper time $\tau=4 \mathrm{fm}$, corresponding to a volume of $V=900 \mathrm{fm}^{3}$. Positions of partons in the transverse direction are taken to have a uniform distribution. Their longitudinal positions are then determined by $z=\tau \sinh y$, as we have assumed that $\eta=y$ due to assumed Bjorken correlation. The resulting total transverse energy per unit rapidity from both expanding quark-gluon plasma and minijet partons is about $590 \mathrm{GeV}$ and is consistent with that measured by the PHENIX collaboration [20]. Most of this transverse energy comes from soft QGP partons as the contribution from minijet partons is only about $10 \%$. The resulting parton density is about $\rho_{\text {parton }} \sim 1 \mathrm{fm}^{-3}$. The thermal parton spectra below the momentum cutoff $p_{0}$ $=2 \mathrm{GeV}$ from the quark-gluon plasma including the collective flow effect are shown in Fig. 1 for gluons (short-dashed curve), $u$ and $d$ (solid curve), $\bar{u}$ and $\bar{d}$ (dashed curve) as well as $s$ and $\bar{s}$ (dash-dotted curve) quarks. Because of scattering of minijet partons with thermal partons as these traverse the quark-gluon plasma, those with momentum around $p_{0}$ are expected to be thermalized with partons in the quark-gluon plasma, leading to a smooth spectrum around $p_{0}$. In the present study, we neglect this effect. 


\section{THE MONTE CARLO METHOD}

In our previous study [6], only partons at midrapidity ( $y$ $=0$ ) are considered. Furthermore, only comoving partons, i.e., partons with momenta in the same transverse direction are allowed to coalesce to hadrons. In these limits, the coalescence formula is reduced to a one-dimensional integral for mesons and a two-dimensional integral for baryons. In the present study, we do not introduce these simplifications. Instead, the multidimensional integrals in the coalescence formula, given by Eqs. (12) and (18), are evaluated by the Monte Carlo method via test particles. Specifically, we introduce a large number of test partons with uniform momentum distribution. To take into account the large difference between numbers of thermal and minijet partons, a test parton with momentum $\mathbf{p}_{\mathrm{T}}$ is given a probability that is proportional to the parton momentum distribution, e.g., $d N_{q} / d^{2} \mathbf{p}_{\mathrm{T}}$ for quarks, with the proportional constant determined by requiring that the sum of all parton probabilities is equal to the parton number. With test partons, the coalescence formulas, Eqs. (12) and (18), are rewritten as

$$
\begin{aligned}
\frac{d N_{M}}{d^{2} \mathbf{p}_{\mathrm{T}}}= & g_{M} \sum_{i, j} P_{q}(i) P_{q}^{-}(j) \delta^{(2)}\left(\mathbf{p}_{\mathrm{T}}-\mathbf{p}_{i \mathrm{~T}}-\mathbf{p}_{j \mathrm{~T}}\right) \\
& \times f_{M}\left(x_{i}, x_{j} ; p_{i}, p_{j}\right)
\end{aligned}
$$

and

$$
\begin{aligned}
\frac{d N_{B}}{d^{2} \mathbf{p}_{\mathrm{T}}}= & g_{B} \sum_{i \neq j \neq k} P_{q}(i) P_{q}(j) P_{q}(k) \delta^{(2)}\left(\mathbf{p}_{\mathrm{T}}-\mathbf{p}_{i \mathrm{~T}}-\mathbf{p}_{j \mathrm{~T}}-\mathbf{p}_{k \mathrm{~T}}\right) \\
& \times f_{B}\left(x_{i}, x_{j}, x_{k} ; p_{i}, p_{j}, p_{k}\right) .
\end{aligned}
$$

In the above, $P_{q}(i)$ and $P_{q}^{-}(j)$ are probabilities carried by $i$ th test quark and $j$ th test antiquark.

The Monte Carlo method introduced here allows us to treat the coalescence of low momentum partons on the same footing as that of high momentum ones. We find that despite a decrease of eight orders of magnitude in real particle spectra, about equal numbers of test hadrons are formed at all momenta.

\section{HADRON TRANSVERSE MOMENTUM SPECTRA}

In this section, we show results for the transverse momentum spectra of pions, antiprotons, and antikaons using the model described in previous sections. For the coalescence contribution, we first take into account the effects due to gluons in the quark-gluon plasma and minijets by converting them to quarks or antiquarks of same momenta, with probabilities according to the flavor compositions in the quarkgluon plasma, as assumed in the ALCOR model [8]. For both mesons and baryons, we include not only coalescence of hard and soft partons as in Ref. [6] but also that among soft as well as hard partons. Furthermore, we include stable hadrons such as pion, nucleon (antinucleon), and kaon (antikaon) as well as unstable resonances such as $\rho, \Delta$, and $K^{*}$. For the latter, these are assumed to be formed with their peak masses. Widths of the resonances are thus not included in evaluating the coalescence probabilities. Since the coalescence model can be viewed as formation of bound states from interacting particles with energy mismatch balanced by other particles in the system, neglecting such off-shell effects is reasonable if the energy mismatch is small. The model is thus applicable for $\rho$ and nucleon (antinucleon) production when we take into account quark masses. For other hadrons, the coalescence probability is expected to be reduced as a result of energy mismatch. The reduction factor can, in principle, be estimated by evaluating the transition probability in the presence of other particles. Since the coalescence radii $\Delta_{x}$ and $\Delta_{p}$ are treated as parameters in our study, the offshell effects can be phenomenologically taken into account by using different coalescence radii for different hadrons. We find that good agreements with experimental data are obtained with coalescence radii $\Delta_{p}=\Delta_{x}^{-1}=0.24 \mathrm{GeV}$ for mesons and $\Delta_{p}=\Delta_{x}^{-1}=0.45 \mathrm{GeV}$ for baryons. We note that there are more $\rho$ and $K^{*}$ than pions and kaons produced from parton coalescence. The pion, proton, and kaon transverse momentum spectra from parton coalescence shown below include contributions from decays of $\rho, K^{*}$, and $\Delta$.

We have also included contributions to hadron production from minijet fragmentations. These are obtained using the KKP fragmentation function [16], which has been shown to reproduce measured high transverse momentum particles at RHIC. Explicitly, hadron momentum spectra are related to minijet parton momentum spectra by

$$
\frac{d N}{d^{2} \mathbf{p}_{\text {had }}}=\sum_{\text {jet }} \int d z \frac{d N}{d^{2} \mathbf{p}_{\text {jet }}} \frac{D_{\text {had } / \mathrm{jet}}\left(z, Q^{2}\right)}{z^{2}},
$$

where $z=p_{\text {jet }} / p_{\text {had }}$ is the fraction of minijet momentum carried by the formed hadron and $Q=p_{\text {had }} / 2 z$ is the momentum scale for the fragmentation process. The KKP fragmentation function is denoted by $D_{\text {had/jet }}\left(z, Q^{2}\right)$.

Since the probability for a minijet parton to coalesce with other partons to form a hadron is much smaller than 1 , independent fragmentation remains the dominant mechanism for hadronization of minijet partons. It is therefore a good approximation to treat minijet fragmentation and coalescence independently without correcting for the competition between the two mechanisms. Although minijet partons have small coalescence probabilities, their contribution to hadrons of intermediate transverse momenta can be more important than that from minijet fragmentations as shown in the results presented in the following sections. This is due to the fact that intermediate transverse momentum hadrons produced through the coalescence process come from minijet partons with lower transverse momentum, while in the fragmentation process these are from minijet partons with higher transverse momentum, as the fragmentation function $D_{\text {had/jet }}\left(z, Q^{2}\right)$ decreases with increasing $z$. As the transverse momentum spectrum of minijet partons is powerlike, their numbers decrease with transverse momentum. The small coalescence probabilities of minijet partons are thus weighted by a larger number of lower transverse momentum minijet partons, leading to a larger contribution to the production of hadrons with inter- 


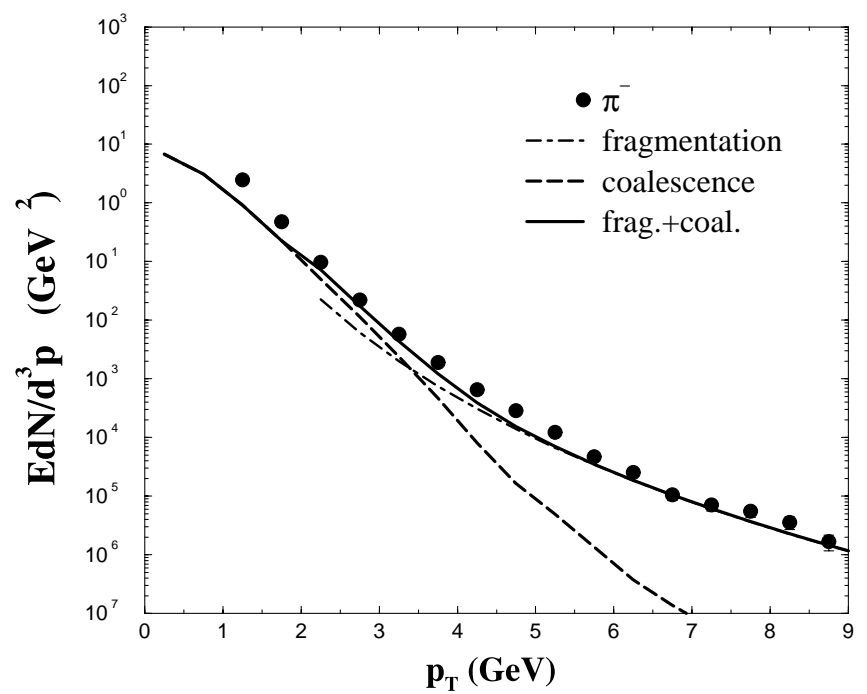

FIG. 2. Pion transverse momentum spectra from $\mathrm{Au}+\mathrm{Au}$ collisions at $\sqrt{s}=200 \mathrm{~A} \mathrm{GeV}$ : direct pion production from parton coalescence (dashed curve); pions from minijet fragmentations (dashdotted curve); and sum from the above two contributions (solid curve). Experimental $\pi^{0}$ data [21] are shown by filled circles.

mediate transverse momenta than that from the fragmentation of higher transverse momentum minijet partons.

Before we show results for hadron spectra and elliptic flows, we would like to point out that the coalescence model as formulated here is applicable if the number of hadrons produced is much less than the parton numbers. In this respect, results for hadrons with momenta above about $1 \mathrm{GeV}$, which account for about $5 \%$ of all partons, are reliable. For lower momentum hadrons, one needs to impose conservation of parton number in converting them to hadrons via coalescence. This correction has not been included in the present study.

\section{A. Pion transverse momentum spectrum}

In Fig. 2, we show the transverse momentum spectrum of pions formed directly from parton coalescence (dashed curve). Pions from fragmentations of minijet partons are shown by the dash-dotted curve. It is seen that pions from parton coalescence dominate low transverse momenta while those from minijet fragmentations are important at high transverse momenta. The two contributions have a similar magnitude at transverse momentum of about $3 \mathrm{GeV}$. Also shown in the figure is the total pion transverse momentum spectrum from the two contributions (solid curve). Compared with measured spectrum from the PHENIX Collaboration [21] (filled circles), the predicted spectrum of the directly produced pion at low transverse momenta is below experimental data, particularly at low transverse momenta.

Since $\rho, K^{*}$, and $\Delta$ decay to pions, we have also included their contributions to the pion transverse momentum spectrum. It is found that $\rho$ meson decays contribute significantly to the pion spectrum at low transverse momenta and bring the final pion spectrum, shown by the solid curve in Fig. 3, in good agreement with experimental data. Also shown in this

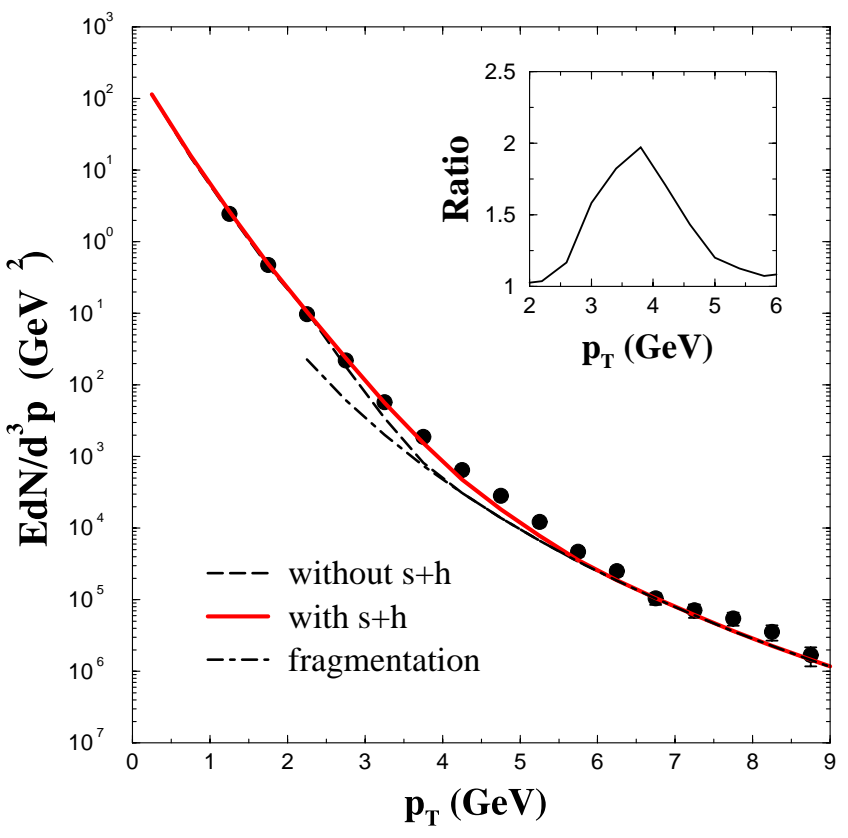

FIG. 3. (Color online) Pion transverse momentum spectra from $\mathrm{Au}+\mathrm{Au}$ collisions at $\sqrt{s}=200 \mathrm{~A} \mathrm{GeV}$ with (solid curve) and without (dashed curve) contributions from coalescence of minijet partons with the quark-gluon plasma partons. Ratio of the two spectra is given in the inset. Experimental $\pi^{0}$ data [21] are shown by filled circles.

figure by the dashed curve is the pion spectrum without including contribution from coalescence of minijet partons with partons in the quark-gluon plasma. It underestimates the pion spectrum at intermediate transverse momenta around 3.5 GeV. The contribution from coalescence of partons from minijets with those from the quark-gluon plasma is more clearly seen from the ratio of pion spectra with and without this contribution, shown in the inset of Fig. 3, which is about a factor of 2 at transverse momenta around $4 \mathrm{GeV}$. Our results thus confirm our previous results in Ref. [6] based on a parametrized pion spectrum from hadronization of the quarkgluon plasma.

\section{B. Antiproton transverse momentum spectrum}

In Fig. 4, we show the antiproton spectrum including those from decays of $\bar{\Delta}$ for $\mathrm{Au}+\mathrm{Au}$ collisions at $\sqrt{s}$ $=200 \mathrm{~A} \mathrm{GeV}$. Results for both with (solid curve) and without (dashed curve) contributions from coalescence of minijet partons with partons from the quark-gluon plasma are shown. These include contributions from fragmentations of minijet partons (dash-dotted curve). The contribution from coalescence of soft and hard partons becomes important when the transverse momentum is above $3 \mathrm{GeV}$, which is somewhat higher than in the case of pion transverse momentum spectrum. Since there are no published experimental data for antiproton transverse momentum spectrum from $\mathrm{Au}+\mathrm{Au}$ collisions at $200 \mathrm{~A} \mathrm{GeV}$, we compare our predictions with the experimental data from the PHENIX collaboration for $\mathrm{Au}+\mathrm{Au}$ collisions at $\sqrt{s}=130 \mathrm{~A} \mathrm{GeV} 4$ [22], shown by filled circles for transverse momenta below 


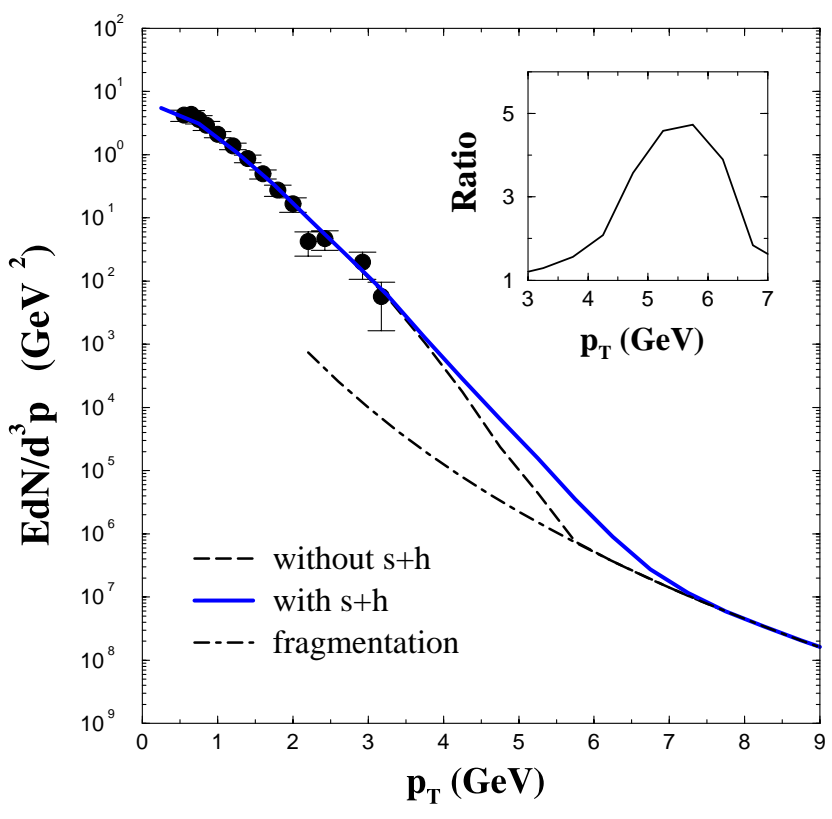

FIG. 4. (Color online) Transverse momentum spectra of antiprotons from $\mathrm{Au}+\mathrm{Au}$ collisions $\sqrt{ } s=200 \mathrm{~A} \mathrm{GeV}$. The dashed curve includes hadrons only from coalescence of partons in the quarkgluon plasma and from independent fragmentations of minijet partons (dash-dotted curve). Adding also hadrons from coalescence of minijet partons with partons in the quark-gluon plasma gives the solid curve. Ratio of the solid to the dashed curve is given in the inset. Experimental $p^{-}$data [22] at $130 \mathrm{~A} \mathrm{GeV}$ are shown by filled circles.

$3 \mathrm{GeV} / c$. Both predictions with and without contributions from coalescence of minijet partons with partons from the quark-gluon plasma are comparable to the experimental data. Shown in the inset of this figure is their ratio as a function of transverse momentum. It is seen that the ratio is close to a factor of 5 at transverse momenta around $5.5 \mathrm{GeV}$. The contribution from coalescence of minijet partons with those from the quark-gluon plasma is thus more important for antiprotons than for pions. It would be of great interest to have experimental data for antiprotons at such high transverse momenta to verify our predictions.

\section{Antiproton to pion ratio}

The antiproton to pion ratio is shown in Fig. 5 as a function of transverse momentum. The solid curve is the result including contributions from both parton coalescence and minijet fragmentations. The ratio increases with transverse momentum up to about $3 \mathrm{GeV} / c$ and decreases with further increasing transverse momentum as in the experimental data [12] shown by filled squares. At high transverse momenta, the antiproton to pion ratio becomes very small as it is largely determined by the results from perturbative QCD (pQCD) [23]. Neglecting the contribution to antiproton production from coalescence of minijet partons with partons from the quark-gluon plasma reduces the antiproton to pion ratio at transverse momenta above $2.5 \mathrm{GeV}$ as shown by the dashed curve in Fig. 5.

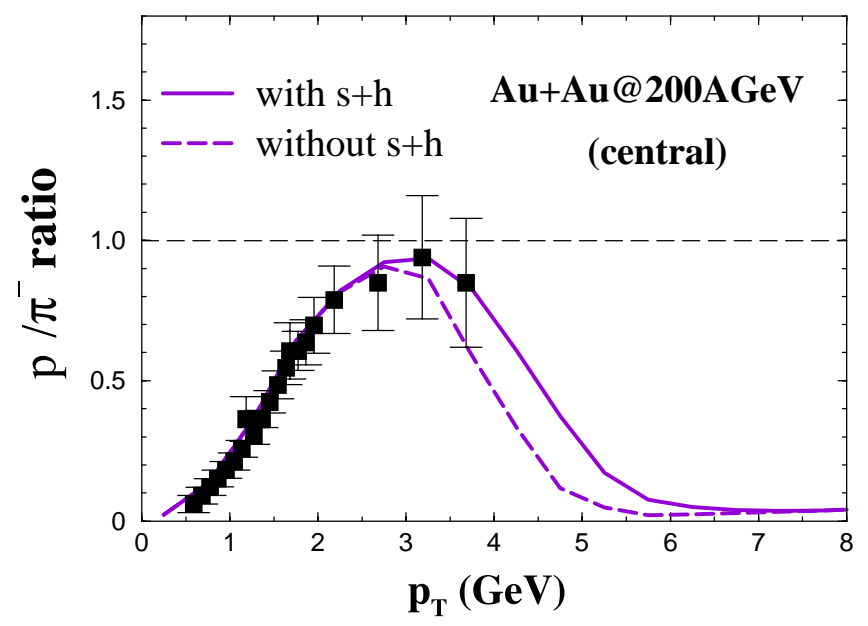

FIG. 5. (Color online) Antiproton to pion ratio from $\mathrm{Au}+\mathrm{Au}$ collisions at $\sqrt{s}=200 \mathrm{~A} \mathrm{GeV}$. Solid and dashed curves are, respectively, results with and without contributions to antiprotons from coalescence of minijet partons with those from the quark-gluon plasma. Filled squares are the experimental data [12].

The enhanced antiproton to pion ratio at intermediate transverse momenta was previously attributed to antiproton production from the baryon junctions in incident nucleons [24]. The possibility of enhanced baryon to pion ratio due to parton coalescence was suggested in Ref. [2]. Using a parton distribution function that is fitted to measured pion transverse momentum spectrum, a parton recombination model similar to the coalescence model indeed leads to a large antiproton to pion ratio at intermediate transverse momenta [4]. In Ref. [5], the antiproton to pion anomaly is explained by the recombination of partons from a quark-gluon plasma with a high effective temperature. Our model further introduces coalescence of minijet partons with partons in the quark-gluon plasma. This makes it possible to account for both the large antiproton to pion ratio at intermediate transverse momenta and its behavior at low transverse momenta.

In our previous work [6], an increasing antiproton to pion ratio at low transverse momenta was also seen, but it was obtained by using different inverse slope parameters for antiproton and pion transverse momentum spectra. In contrast, results obtained in the present work are from coalescence of soft partons in the quark-gluon plasma.

\section{Antikaon transverse momentum spectrum and antikaon to pion ratio}

In Fig. 6, we show the antikaon spectrum including those from decays of $K^{*-}$ for $\mathrm{Au}+\mathrm{Au}$ collisions at $\sqrt{s}$ $=200 \mathrm{~A} \mathrm{GeV}$. Results including antikaon production from coalescence of soft partons from the quark-gluon plasma are shown by the dashed curve while those including also coalescence between minijet partons with partons in the quarkgluon plasma are shown by the solid curve. Contributions from minijet fragmentations (dash-dotted curve) are also included. As for antiprotons, we compare these predictions with the experimental data shown by filled squares from the PHENIX collaboration for $\mathrm{Au}+\mathrm{Au}$ collisions at $\sqrt{s}$ $=130 \mathrm{~A} \mathrm{GeV}[22]$, as there are no published data at $\sqrt{s}$ 


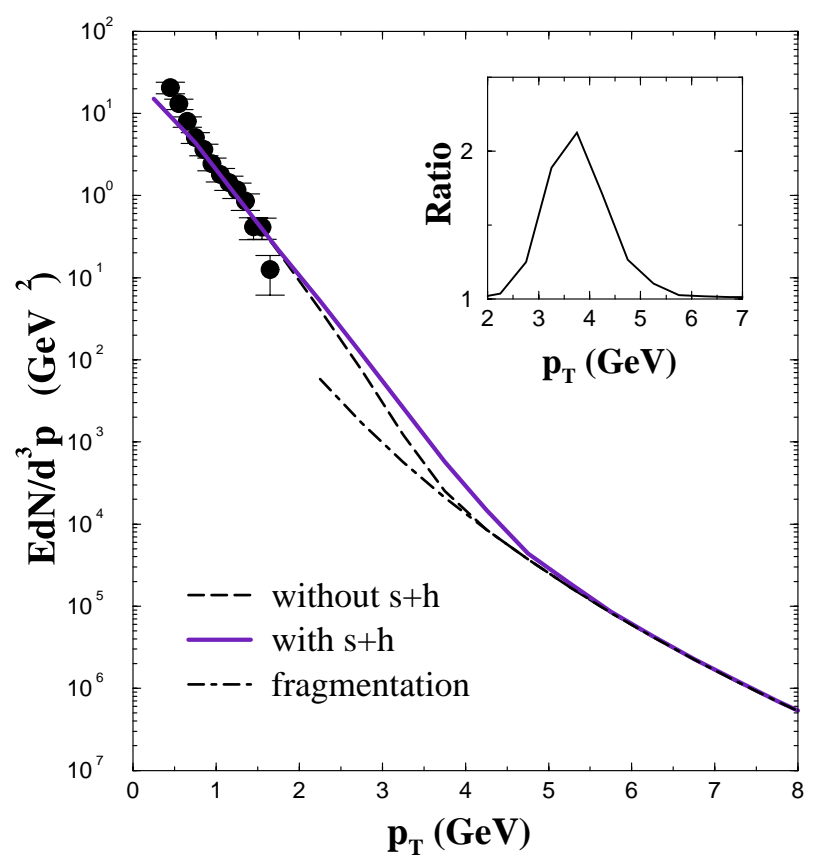

FIG. 6. (Color online) Transverse momentum spectra of $K^{-}$ from $\mathrm{Au}+\mathrm{Au}$ collisions at $\sqrt{s}=200 \mathrm{~A} \mathrm{GeV}$. The dashed curve includes contributions only from coalescence of partons in the quarkgluon plasma and hadrons from independent fragmentations of minijet partons (dash-dotted curve). Adding also hadrons from coalescence of minijet partons with partons in the quark-gluon plasma gives the solid curve. Ratio of the solid to the dashed curve is given in the inset. Experimental $K^{-}$data [22] at $130 \mathrm{~A} \mathrm{GeV}$ are shown by filled circles.

$=200 \mathrm{~A} \mathrm{GeV}$. For the limited data below $2 \mathrm{GeV}$, the coalescence model reproduces them very well without the contribution from coalescence of minijet partons with partons from the quark-gluon plasma as the latter becomes important when the transverse momentum is above $2.5 \mathrm{GeV}$. Shown in the inset of Fig. 6 is the ratio of the predictions with and without the contribution from soft and hard parton coalescence as a function of transverse momentum. The ratio reaches more than 2 at transverse momenta around $4 \mathrm{GeV}$. The contribution to antikaons from coalescence of minijet partons with those from the quark-gluon plasma is thus comparable to that for pions.

The antikaon to pion ratio is shown in Fig. 7 as a function of transverse momentum. The solid curve is the result including contributions from both parton coalescence and minijet fragmentations. For transverse momenta below about $2 \mathrm{GeV}$, this ratio is similar to the antiproton to pion ratio except that its value is smaller. At higher transverse momenta, the antikaon to pion ratio decreases slightly and reaches the value predicted by pQCD at high transverse momenta [25], which gives a larger antikaon to pion ratio than the antiproton to pion ratio. Results without contribution to antikaon production from coalescence of minijet partons with partons from the quark-gluon plasma are given by the dashed curve, and it gives a smaller antikaon to pion ratio at intermediate transverse momenta compared to that with this contribution. Our results thus demonstrate again the importance of antikaon

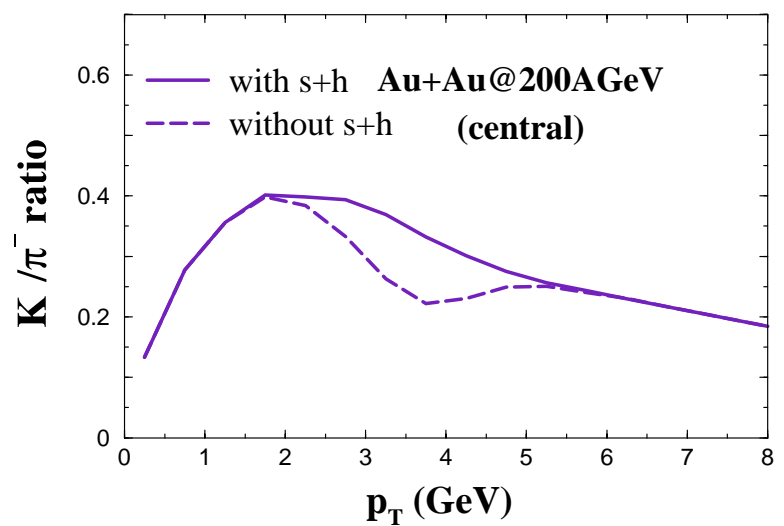

FIG. 7. (Color online) Antikaon to pion ratio from $\mathrm{Au}+\mathrm{Au}$ collisions at $\sqrt{s}=200 \mathrm{~A} \mathrm{GeV}$. Solid and dashed curves are, respectively, results with and without contributions to antikaon production from coalescence of minijet partons with those from the quarkgluon plasma.

production from coalescence of partons from minijets and quark-gluon plasma.

\section{TRANSVERSE FLOW EFFECT}

It is interesting to study the effect of transverse flow of quark-gluon plasma on the transverse momentum spectra of produced hadrons. In fact, the increase of antiproton to pion ratio at low transverse momenta was believed to be due to a stronger transverse flow effect on protons than on pions [26]. Such effect was assumed in our previous study [6] by using a larger inverse slope parameter for the antiproton transverse momentum spectrum than that for pions. In the present model, soft hadrons are produced from coalescence of partons in the quark-gluon plasma, which is given a collective flow velocity of $0.5 c$, and the resulting antiproton to pion ratio at low transverse momentum is found to increase with transverse momentum. To see if this is due to the collective flow effect introduced in the model, we have repeated the calculations without transverse flow effect on antiprotons. These results, which include only contribution to antiproton production from coalescence of partons in the quark-gluon plasma, are shown in Fig. 8 by dashed curves for pions (upper panel) and antiprotons (lower panel). Compared with results obtained with a collective flow velocity of $0.5 c$, shown by solid curves in Fig. 8, collective flow affects the pion and antiproton spectra mainly at transverse momenta above 1.5 $\mathrm{GeV}$, and its effect is stronger for antiprotons than for pions. The reason that for low transverse momenta the inverse slope parameter for pions remains smaller than that for antiprotons in the absence of collective flow is due to the fact that most low transverse momentum pions are from decays of $\rho$ mesons, which gives a smaller effective slope parameter for pions than that of directly produced pions. Our results thus show that the increase seen in the antiproton to pion ratio at low transverse momenta is not necessarily due to the collective flow of quark-gluon plasma.

Since collective flow affects hadron spectra at intermediate transverse momenta as shown in Fig. 8 and its insets, where the ratio of the pion or antiproton spectrum obtained 


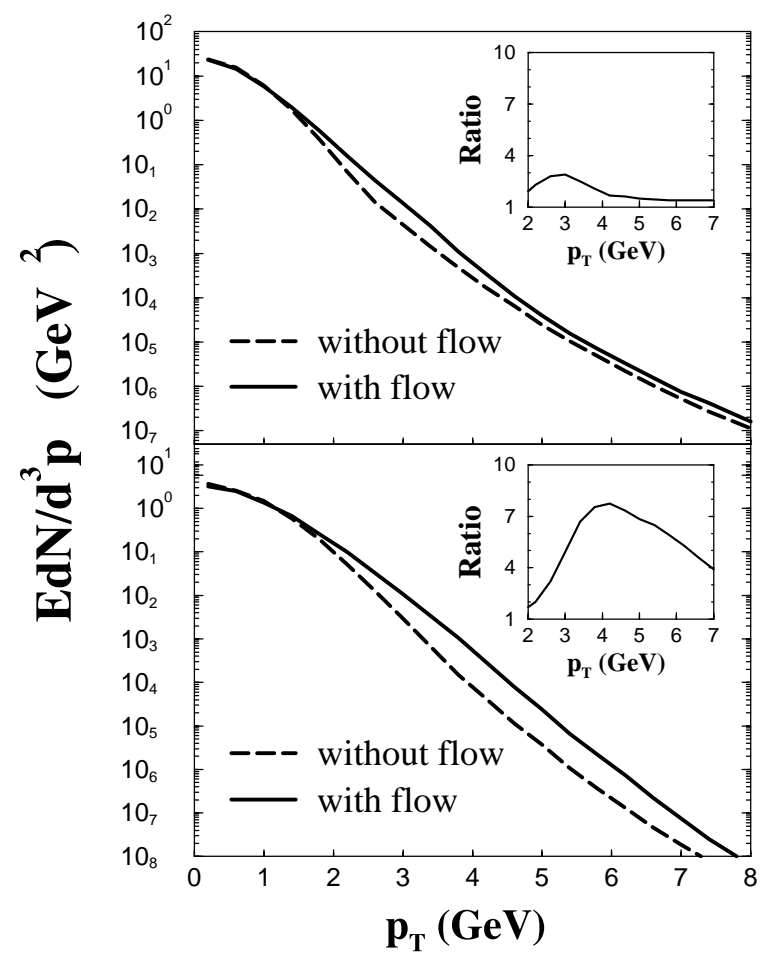

FIG. 8. Pion (upper panel) and antiproton (lower panel) transverse momentum spectra from coalescence of partons in the quarkgluon plasma with (solid curves) and without (dashed curves) collective transverse flow in the quark-gluon plasma. Ratios of these two results are shown in the insets.

with and without collective flow in the quark-gluon plasma is shown, it is expected to have a large effect on the antiproton to pion ratio at these momenta. This is shown in Fig. 9, where the antiproton to pion ratio is given for both with (solid curve) and without (dashed curve) collective flow effect on protons. It is seen that collective flow enhances significantly this ratio for transverse momenta between 2 and $5 \mathrm{GeV} / c$. The collective flow effect on intermediate transverse momentum antiprotons is thus as strong as the effect due to coalescence of partons from minijets with those from

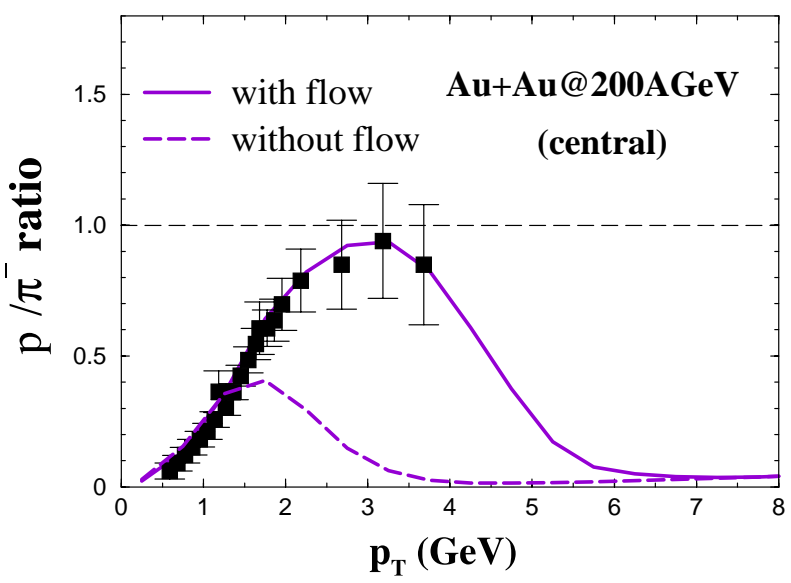

FIG. 9. (Color online) Antiproton to pion ratio with (solid curve) and without (dashed curve) collective flow in the quark-gluon plasma. Experimental data are shown by filled squares. the quark-gluon plasma, shown in Fig. 5. To confirm the mechanism for antiproton production from coalescence of minijet and quark-gluon plasma partons, it is thus important to have a quantitative understanding of collective flow in the quark-gluon plasma.

\section{ELLIPTIC FLOWS}

The parton coalescence model based on the test particle Monte Carlo method is also useful for studying other observables at RHIC. One problem that can be addressed with this model is to study how elliptic flows of hadrons are related to that of partons $[3,27]$. We carry out such a study following the idea of Ref. [4], where the parton transverse momentum distribution is extracted from fitting the pion transverse momentum spectrum using the quark recombination or coalescence model and is then used to predict that of antiprotons. Here, we extract the elliptic flows of light and strange quarks by fitting the measured pion and kaon elliptic flows based on the coalescence model. The resulting quark elliptic flows are then used to predict the elliptic flows of protons, $\Lambda, \Xi, \Omega$, and $\phi$ mesons.

The elliptic flow is a measure of the anisotropy of the particle transverse momentum spectrum, i.e.,

$$
v_{2}=\left\langle\frac{p_{x}^{2}-p_{y}^{2}}{p_{x}^{2}+p_{y}^{2}}\right\rangle,
$$

where the transverse axes $x$ and $y$ are, respectively, in and out of the reaction plane. Including transverse momentum anisotropy, the quark transverse momentum distribution is given by

$$
\frac{d N_{q}}{d^{2} \mathbf{p}_{\mathrm{T}}}=\frac{d N_{q}}{p_{\mathrm{T}} d p_{\mathrm{T}} d \phi}=\frac{d N_{q}}{p_{\mathrm{T}} d p_{\mathrm{T}}}\left[1+2 v_{2} \cos (2 \phi)\right],
$$

where $\phi$ is the azimuthal angle in the transverse plane.

In Fig. 10, we show by a dotted curve the elliptic flows of light quarks and antiquarks together with the pion elliptic flow shown by a dashed curve, which is supposed to reproduce the measured pion elliptic flow given by filled squares [28]. The predicted proton elliptic flow obtained from the quark and antiquark elliptic flows is then given by the dashed curve and is seen to agree with that measured in experiments shown by filled circles [28].

The elliptic flow of strange hadrons is shown in Fig. 11. The strange quark and antiquark elliptic flow (dotted curve) is obtained by fitting the calculated Kaon elliptic flow (dashed curve) to the measured on (filled squares) [29]. The predicted $\Lambda$ elliptic flow shown by the dashed curve is seen to agree with the available experimental data [29] given by filled circles. We have also predicted the $\phi$ meson (dashed curve with stars), $\Xi$ (solid curve with stars), and $\Omega$ elliptic flows (dash-dotted curve). It is seen that due to a smaller (about 30\%) strange quark elliptic flow than that of light quarks, the $\phi$ meson elliptic flow is smaller than that of kaons, while the $\Omega$ elliptic flow is smaller than that of $\Xi$, which is further smaller than that of $\Lambda$. For all hadrons considered here, decrease of their elliptic flows at high trans- 


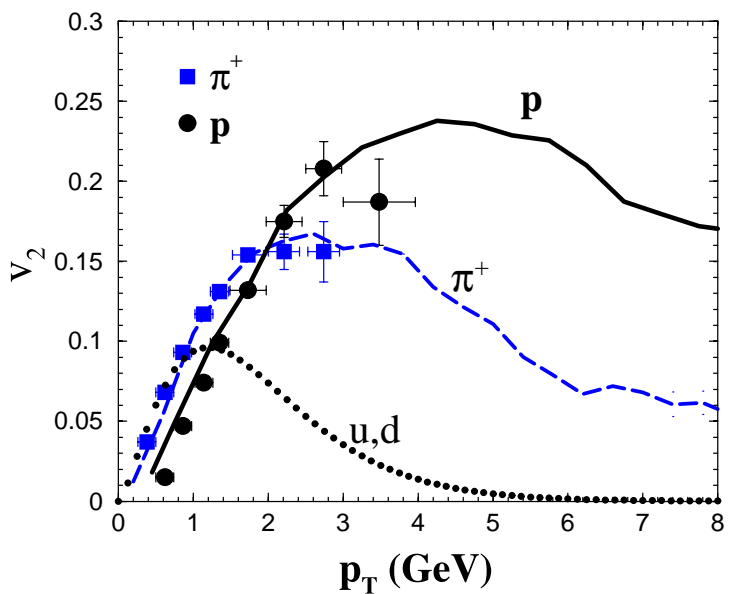

FIG. 10. (Color online) Elliptic flows of pions (dashed curve) and protons (solid curve) as functions of transverse momentum. Elliptic flow of light quarks and antiquarks is given by the dotted curve. Experimental data [28] are shown by filled squares for pions and filled circles for protons.

verse momenta is due to our assumption that high transverse momentum minijet partons and hadrons from minijet fragmentations have vanishing elliptic flows. Of course, if high transverse momentum minijet partons also develop elliptic flows as suggested in Ref. [30], our results on hadron elliptic flows at high transverse momenta should be modified.

\section{SUMMARY AND OUTLOOK}

In this paper, we have studied the hadronization of quarkgluon plasma and minijet partons produced in relativistic heavy ion collisions in terms of the parton coalescence model. The momentum spectra of partons in the quark-gluon plasma are taken to have an exponential form with an inverse slope parameter similar to the phase transition temperature, while partons in the minijets have power-law spectra. The

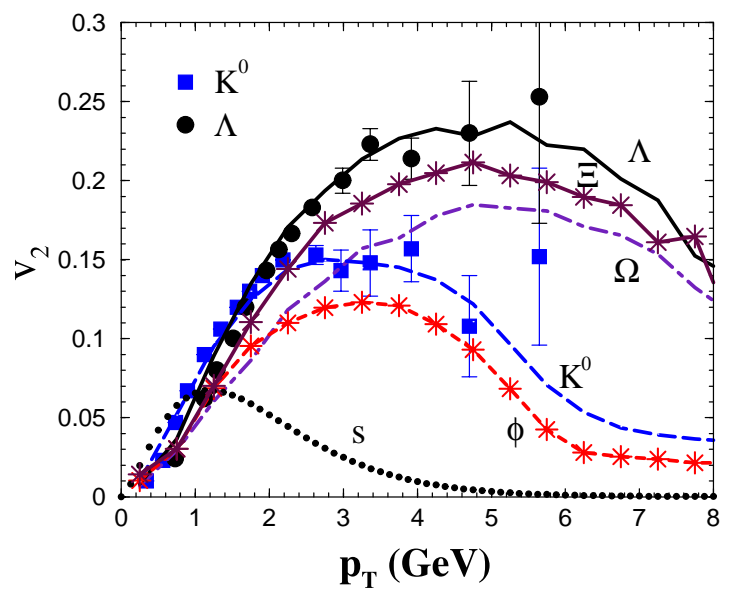

FIG. 11. (Color online) Elliptic flows of kaons (solid curve), $\phi$ mesons (dashed curve with stars), $\Lambda$ (dashed curve), $\Xi$ (solid curve with stars), and $\Omega$ (dash-dotted curve). Elliptic flow of strange quarks and antiquarks is given by the dotted curve. Experimental data [29] are shown by filled squares for kaons and circles for $\Lambda$. flavor compositions in the quark-gluon plasma are determined by the experimentally measured antiproton to proton ratio and strange to nonstrange particle ratios. The volume of the quark-gluon plasma is then fixed by the total transverse energy measured in experiments. A collective flow is introduced in the quark-gluon plasma with a flow velocity comparable to that extracted from experiments. To take into account the vast difference in the magnitude of the minijet parton transverse momentum spectrum and that of partons in the quark-gluon plasma, a test particle Monte Carlo method has been introduced to efficiently evaluate the coalescence probability of partons. Both soft partons from the quarkgluon plasma and hard partons from minijets are used in the coalescence model to produce hadrons such as pions, kaons (antikaons), $\rho$ mesons, $K^{*}$ 's, nucleons (antinucleons), and $\Delta$ $(\bar{\Delta})$ resonances. Specifically, we have included coalescence of hard minijet partons with soft partons besides that among soft and hard partons.

The resulting pion, antikaon, and antiproton spectra are seen to agree with available experimental data from RHIC. For pions, contributions from $\rho$ decays are important in explaining the measured transverse momentum spectrum below $2 \mathrm{GeV}$. For intermediate transverse momentum spectra of pions and antikaons between 2 and $5 \mathrm{GeV}$, including contributions from coalescence of minijet partons with those from the quark-gluon plasma are important, leading to a factor of 2 enhancement compared to results without this contribution. This effect is even larger for antiprotons, where the enhancement reaches a factor of 5 at transverse momenta around 5 $\mathrm{GeV}$. We have also compared the transverse momentum dependence of antiproton to pion ratio to the experimental data. Results from the coalescence model are found to agree quite well with the available data, i.e., it increases with transverse momentum and reaches a value of about 1 at transverse momentum of about $3 \mathrm{GeV}$. With further increase in transverse momentum, our model predicts that the antiproton to pion ratio should decrease and approach the small value given by the $\mathrm{pQCD}$. We have also calculated the antikaon to pion ratio as a function of transverse momentum. The result is similar to that for antiproton to pion ratio but with a smaller magnitude and a larger value at high transverse momenta. We further find that these ratios are reduced if antiproton production from coalescence of minijet partons with partons in the quark-gluon plasma is neglected. This effect is, however, comparable to that due to collective flow of the quark-gluon plasma. To confirm the mechanism for antiproton production from coalescence of minijet partons with partons in the quark-gluon plasma thus requires a quantitative understanding of collective flow effects in the quark-gluon plasma.

We have also studied elliptic flows of hadrons using quark and antiquark elliptic flows fitted to the measured pion and kaon elliptic flows. Predicted proton and $\Lambda$ elliptic flows agree with those measured in experiments. We have further predicted the elliptic flows of $\phi$ mesons, $\Xi$, and $\Omega$, and they are smaller than those of nonstrange hadrons due to smaller strange quark elliptic flow than that of light quarks.

The parton coalescence model based on the test particle Monte Carlo method can be extended to include collision dynamics of partons and hadrons using parton [31] and had- 
ron [32] transport models. This would make it possible to study effects due to final-state hadronic scattering on the transverse momentum spectra and elliptic flows of hadrons, which have been neglected in the present study as we have compared hadron spectra and elliptic flows from parton coalescence directly with experimental data. Also, including parton scatterings would allow us to treat properly the parton spectrum around the cutoff momentum $p_{0}$, resulting in a smooth spectrum at this momentum. It further makes it possible to determine both transverse and elliptic flows of partons from the collision dynamics instead of treating them as input as in the present study. Moreover, including expansion dynamics of the system will ensure that the total entropy does not decrease even though the entropy density is reduced when hadrons are formed from coalescence of partons.

\section{ACKNOWLEDGMENTS}

We thank Su Houng Lee for helpful discussions. This paper was based on work supported by the U.S. National Science Foundation under Grant No. PHY-0098805 and the Welch Foundation under Grant No. A-1358. V.G. was also supported by the National Institute of Nuclear Physics (INFN) in Italy, while P.L. was supported by the Hungarian OTKA Grant Nos. T034269 and T043455. P.L. further thanks G. Papp and G. Fai for discussions on pQCD results.
[1] Z.W. Lin and C.M. Ko, Phys. Rev. C 65, 034904 (2002); Z.W. Lin, C.M. Ko, and S. Pal, Phys. Rev. Lett. 89, 152301 (2002).

[2] S.A. Voloshin, Nucl. Phys. A715, 379 (2003).

[3] D. Molnar and S.A. Voloshin, nucl-th/0302014.

[4] R.C. Hwa and C.B. Yang, Phys. Rev. C 67, 034902 (2003); Phys. Rev. Lett. 90, 212301 (2003); Phys. Rev. C 67, 064902 (2003).

[5] R.J. Fries, B. Müller, C. Nonaka, and S.A. Bass, Phys. Rev. Lett. 90, 202303 (2003).

[6] V. Greco, C.M. Ko, and P. Lévai, Phys. Rev. Lett. 90, 202302 (2003).

[7] Z.W. Lin and C.M. Ko, Phys. Rev. Lett. 89, 202302 (2002).

[8] T.S. Biró, P. Lévai, and J. Zimányi, Phys. Lett. B 347, 6 (1995); Phys. Rev. C 59, 1574 (1999); T.S. Biró, T. Csörgö, P. Lévai, and J. Zimányi, Phys. Lett. B 472, 243 (2002).

[9] P. Csizmadia and P. Lévai, Phys. Rev. C 61, 031903 (2000); J. Phys. G 28, 1997 (2002).

[10] B. Zhang, C.M. Ko, B.A. Li, and Z. Lin, Phys. Rev. C 61, 067901 (2000); B. Zhang, C.M. Ko, B.A. Li, Z. Lin, and B.H. Sa, ibid. 62, 054905 (2000); B. Zhang, C.M. Ko, B.A. Li, Z.W. Lin, and S. Pal, ibid. 65, 054909 (2002); Z.W. Lin, S. Pal, C.M. Ko, B.A. Li, and B. Zhang, ibid. 64, 011902 (2001); Nucl. Phys. A498, 375c (2002).

[11] X.N. Wang, Phys. Rev. C 58, 2321 (1998).

[12] K. Adcox et al., PHENIX Collaboration, Phys. Rev. Lett. 88, 242301 (2002); T. Chujo, Nucl. Phys. A715, 151 (2003); T. Sakaguchi, ibid. A715, 757 (2003).

[13] C. Dover, U. Heinz, E. Schnedermann, and J. Zimányi, Phys. Rev. C 44, 1636 (1991).

[14] Y. Zhang et al., Phys. Rev. C 65, 034903 (2002).
[15] M. Glück, E. Reya, and A. Vogt, Z. Phys. C 67, 433 (1995).

[16] B.A. Kniehl, G. Kramer, and B. Pötter, Nucl. Phys. B582, 514 (2000); B597, 337 (2001).

[17] P. Lévai et al., Nucl. Phys. A698, 631 (2002); M. Gyulassy, P. Lévai, and I. Vitev, Phys. Lett. B 538, 282 (2002).

[18] M. Gyulassy, P. Lévai, and I. Vitev, Phys. Rev. Lett. 85, 5535 (2000); Nucl. Phys. B571, 197 (2000); B594, 371 (2001); M. Gyulassy, I. Vitev, and X.N. Wang, Phys. Rev. Lett. 86, 2537 (2001).

[19] P. Lévai and U. Heinz, Phys. Rev. C 57, 1879 (1998).

[20] K. Adcox et al., PHENIX Collaboration, Phys. Rev. Lett. 87, 052301 (2001).

[21] S.S. Adler et al., PHENIX Collaboration, nucl-ex/0304022.

[22] K. Adcox et al., PHENIX Collaboration, Phys. Rev. Lett. 88, 022301 (2002).

[23] X. Zhang, G. Fai, and P. Lévai, Phys. Rev. Lett. 89, 272301 (2002).

[24] I. Vitev and M. Gyulassy, Phys. Rev. C 65, 041902 (2002).

[25] P. Lévai, G. Papp, G. Fai, and M. Gyulassy, nucl-th/0012017.

[26] U. Heinz, talk presented at PANIC02, nucl-th/0212004.

[27] Z.W. Lin and D. Molnar, nucl-th/0304045.

[28] S. Esumi, Nucl. Phys. A715, 599 (2003).

[29] R. Snellings, for STAR Collaboration, 19th Winter Workshop on Nuclear Dynamics, nucl-ex/0305001; P. Sorensen, for STAR Collaboration, Strange Quark Matter 2003, nucl-ex/0305008.

[30] M. Gyulassy, I. Vitev, and X.N. Wang, Phys. Rev. Lett. 86, 2537 (2001); Phys. Lett. B 526, 301 (2002).

[31] B. Zhang, Comput. Phys. Commun. 109, 193 (1998).

[32] B.A. Li and C.M. Ko, Phys. Rev. C 52, 2037 (1995). 